\title{
Comparative study of fully 3D reconstruction algorithms for lens-free microscopy
}

\author{
Anthony Berdeu ${ }^{1,2,}{ }^{*}$, Fabien Momey ${ }^{3, *}$, Bastien Laperrousaz ${ }^{1,4,5}$, Thomas Bordy ${ }^{1,2}$, Xavier \\ Gidrol $^{1,4,5}$, Jean-Marc Dinten ${ }^{1,2}$, Nathalie Picollet-D’hahan ${ }^{1,4,5}$, And CÉdric Allier ${ }^{1,2}$ \\ ${ }^{1}$ Univ. Grenoble Alpes, F-38000 Grenoble, France \\ ${ }^{2}$ CEA, LETI, MINATEC Campus, F-38054 Grenoble, France \\ ${ }^{3}$ Laboratoire Hubert Curien - Univ. Jean Monnet, F-42100 Saint-Étienne, France \\ ${ }^{4}$ CEA, BIG, Biologie à Grande Echelle, F-38054 Grenoble, France \\ ${ }^{5}$ INSERM, U1038, F-38054 Grenoble, France \\ *Corresponding authors: anthony.berdeu@cea.fr, fabien.momey@univ-st-etienne.fr
}

Compiled August 22, 2019

\begin{abstract}
We propose a 3D imaging platform based on lens-free microscopy to perform multi-angle acquisitions on 3D cell cultures embedded in extracellular matrices. Lens-free microscopy acquisitions present some inherent issues such as the lack of phase information on the sensor plane and a limited angular coverage. We developed and compared three different algorithms based on the Fourier diffraction theorem to obtain fully 3D reconstructions. These algorithms present an increasing complexity associated with a better reconstruction quality. Two of them are based on a regularised inverse problem approach. To compare the reconstruction methods in terms of artefacts reduction, signal-to-noise ratio and computation time, we tested them on two experimental datasets: an endothelial cell culture and a prostate cell culture grown in a 3D extracellular matrix with large reconstructed volumes up to $\sim 5 \mathrm{~mm}^{3}$ with a resolution sufficient to resolve isolated single cells. The lens-free reconstructions compare well with standard microscopy.
\end{abstract}

OCIS codes: (090.1970) Diffractive optics; (090.1995) Digital holography; (100.3010) Image reconstruction techniques; (170.0110) Imaging systems; (180.6900) Three-dimensional microscopy.

http://dx.doi.org/10.1364/AO.56.003939

\section{INTRODUCTION}

The study of in-vitro cell populations is a challenge if one wants to gather quantitative and systematic data over extended periods of time on a large volume while preserving the integrity of the living sample. As stated in [1], the solution "requires our microscopes to become smart and gentle."

Lens-free time-lapse microscopy provides a simple, cheap and robust tool meeting these needs in the realm of $2 \mathrm{D}$ cell culture [2-4]. It is label-free and non-phototoxic and allows the analyse of biological samples at different scales both in space and time. Its minimal and compact design is adapted to incubator conditions. It allows the study of cell cultures on large periods of time without the toxicity induced by classical staining and labelling methods. It offers a large field of view equal to the sensor surface (a few tens of squared millimetres for standard CMOS sensors). This gives the opportunity to study populations of up to twenty thousand cells at the same time with a resolution, strongly linked to the pixel pitch, sufficient to individually distinguish every single cell [2].

Unlike conventional 2D cell culture systems, 3D cultures al- low to study biological processes closer to physiological reality such as tissue morphogenesis or tumour initiation [5-7]. Along with the standardisation of 3D culture protocols in cell biology, lens-free imaging techniques must also be extended to this new modality both in terms of acquisition methods and 3D reconstruction algorithms for large organoid structures [8-11].

A previous work [11] introduced a method for 3D lens-free tomography for large 3D biological samples but implied various artefacts. To improve both the acquisitions and reconstruction methods, we present here a new design enhancing the angular coverage of the object. Based on this new imaging device, we tested and compared new algorithms to overcome the two pitfalls of lens-free tomographic acquisitions: the lack of phase information in the in-line holographic configuration and the limited angular coverage. We designed three fully 3D reconstruction algorithms, all based on the Fourier diffraction theorem as used in standard diffractive tomography [12-15]. They leads to an increasing reconstruction quality at the cost of increased complexity:

- The first method C.1, developed in [11], reintroduces an 
approximation of the phase by a phase ramp in the dataset.

- The second algorithm C.2 performs an iterative phase retrieval based on a regularised inverse problem approach on each 2D acquisition. In comparison to the former method, this algorithms handles better the lack of phase information in the data acquisition process by estimating a realistic guess for it.

- The third solution C.3 also uses the regularised inverse problem formalism, but directly applied on the whole dataset and the 3D object to retrieve, the Fourier diffraction theorem providing the direct model. Doing so, at the cost of an increased computation time, we aim to overcome the limited number of acquisitions and admissible angles around the sample by directly adding constraints on the 3D object. To our knowledge, this is the first time that such an inverse problem approach is implemented in the context of lens-free diffractive tomography which enables the reconstruction of large volumes of unstained biological samples.

To compare the reconstruction methods in terms of artefacts reduction, signal-to-noise ratio and computation time, we tested them on two experimental datasets: a HUVEC endothelial cell culture and a RWPE1 prostate cell culture grown in a 3D extracellular matrix of Matrigel ${ }^{\circledR}$. The first one presents a planar geometry and the second one is a complex fully 3D structure.

In the following, we first introduce the experimental bench we developed dedicated to lens-free diffractive tomography of 3D biological samples. Next, we present the Fourier diffraction theorem and the three dedicated reconstruction algorithms we developed to retrieve 3D objects with our lens-free microscope. We then compare the $3 \mathrm{D}$ reconstructions of the two above mentioned datasets to estimate the performances of the three proposed reconstruction methods. Reconstructed volumes as large as $\sim 5 \mathrm{~mm}^{3}$ are presented with a resolution sufficient to resolve isolated single cells. We conclude with a comparison with phase contrast and fluorescent microscopy.

\section{MATERIALS AND METHODS}

\section{Experimental setup}

Standard 2D lens-free microscopes are minimalist tools, only composed of a semi-coherent illumination source fixed above a sensor [2-4]. It is based on the in-line holography principle (see figure 1) proposed by Gabor in 1948 [16]. The 2D cell culture is placed on the top of the sensor at a distance of 1 to $3 \mathrm{~mm}$ and diffracts the normally incident light. The object is then numerically retrieved from the recorded hologram.

We developed an experimental bench to adapt this geometry for the reconstruction of 3D objects which requires to multiply the viewing angles (see figure 1). The object is static while the sensor ${ }^{1}$ and the multi-wavelength illumination source ${ }^{2}$ are rotating around an axis orthogonal to the sensor with a stepper motor $^{3}$.

Unlike the 2D in-line geometry, the illumination is tilted by an angle $\theta=45^{\circ}$ and the sensor is slightly deported so that the hologram of the 3D object remains centered regardless of the position of its geometrical projection according to the angle $\varphi$ around the 3D sample. This allows to optimise the 2D field of

${ }^{1}$ CMOS sensor - IDS - $29.4 \mathrm{~mm}^{2}, 3840 \times 2748$ monochromatic pixels, pixel pitch $1.67 \mu \mathrm{m}-$ ref. UI-1942LE-M

${ }^{2}$ LED CREE, $\lambda \in\{450,520,630 \mathrm{~nm}\}-$ ref. XLamp MC-E RGBW MCE4CT

${ }^{3}$ RS-535-0401, 0.9, 44 Ncm, $2.8 \mathrm{~V}, 1.68$ A, 4 Wires

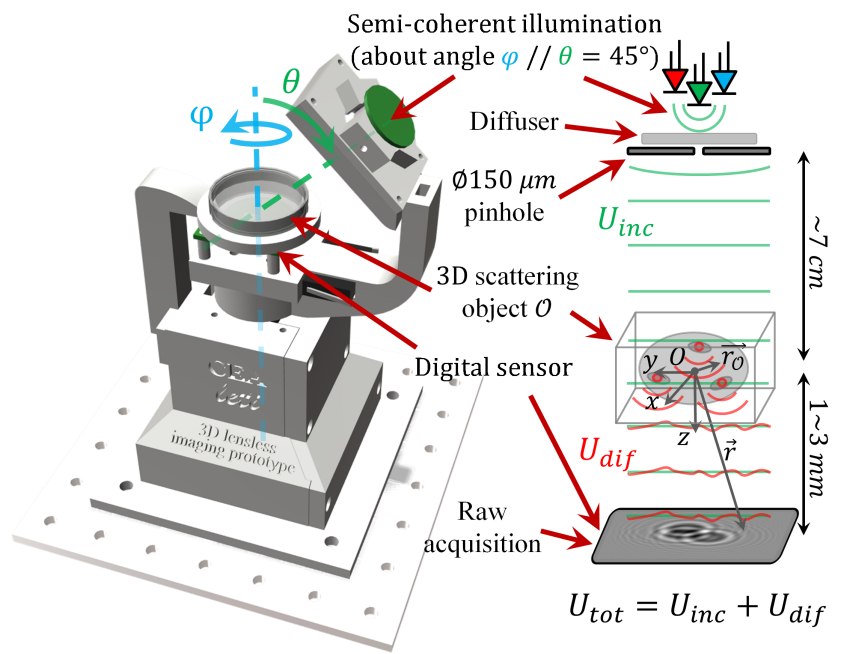

Fig. 1. Left - Experimental setup dedicated to lens-free diffractive tomography. Right - Optical scheme of the system. The semicoherent incident plane wave $U_{i n c}$ is scattered by the $3 \mathrm{D}$ sample. Each element of the volume behaves like a secondary spherical source which creates a diffracted wave $U_{d i f}$. The sensor records the intensity of their sum: $I_{t o t}=\left|U_{t o t}\right|^{2}$ with $U_{t o t}=U_{\text {inc }}+U_{\text {dif }}$.

view of the sensor, which increases the overall volume that can be reconstructed. This configuration is well adapted to standard culture cell containers such as Petri dishes.

\section{D diffraction physics}

The absence of reference arm as in off-axis holography for the incident wave in the device presented on figure 1 implies that only the intensity $I_{t o t}$ of the complex total wave $U_{t o t}$ is recorded by the sensor. This wave is the summation of the incident plane wave $U_{i n c}$ produced by the LED and the diffracted wave $U_{d i f}$ scattered by the object:

$$
I_{t o t}=\left|U_{t o t}\right|^{2}=\left|U_{i n c}+U_{d i f}\right|^{2}
$$

The input signal is composed of intensities at different illumination positions and wavelengths. From this, we have to retrieve the 3D diffracting sample. The latter is described by its scattering potential $f$ defined at each point of space $\vec{r}=(x, y, z)$ by:

$$
f(\vec{r})=\left(\left(\frac{n(\vec{r})}{n_{0}}\right)^{2}-1\right)
$$

where $n(\vec{r})$ is the local complex refractive index and $n_{0}$ is the refractive index of the surrounding medium. In the hypothesis of transparent objects, the scattering potential is supposed to be the same regardless of the illumination wavelength.

Under the first-order Born approximation [12], the wave $U_{d i f}$ diffracted by the object $\mathcal{O}$ (see figure 1) can be expressed in terms of $f$ :

$$
U_{d i f}(\vec{r}) \simeq \frac{k_{0}^{2}}{4 \pi} \iiint_{\overrightarrow{r_{\mathcal{O}}} \in \mathcal{O}} f\left(\overrightarrow{r_{\mathcal{O}}}\right) U_{i n c}\left(\overrightarrow{r_{\mathcal{O}}}\right) \frac{e^{i k_{0}\left\|\vec{r}-\overrightarrow{r_{\mathcal{O}}}\right\|}}{\left\|\vec{r}-\overrightarrow{r_{\mathcal{O}}}\right\|} d^{3} \overrightarrow{r_{\mathcal{O}}}
$$

with $k_{0}=2 \pi n_{0} / \lambda$ the wave number at a given illumination wavelength $\lambda$ in a medium of refractive index $n_{0}$. This convolution highlights the 3D Huygens-Fresnel principle which states 
that the diffracted wave is the interference of all the secondary spherical waves produced by each point of the sample weighted by the incident wave and the local scattering potential.

This equation can consequently be used to retrieve the scattering potential $f$ from the knowledge of the diffracted wave $U_{\text {dif }}$. From equation 2, it is then possible to get the 3D map of the complex refractive index of the 3D sample. Its real part is the standard refractive index while its imaginary part represents the object absorption [17, 18].

Nevertheless, the Born approximation holds only if the overall phase delay introduced by the sample remains negligible [13], a requirement which is generally not met in cell cultures that contain objects with dimensions larger than few tens of microns. For a cell with a typical length of $l=20 \mu \mathrm{m}$ and refractive index of 1.36 in water $[13,19]$, the relative refractive index is $\delta n=0.03$. In the visible light $\lambda \simeq 550 \mathrm{~nm}$ the introduced phase delay is $2 \pi l \delta n / \lambda>2 \pi$, which cannot be considered negligible. While this prevents any quantitative reconstruction, we will however use the model described by equation (3) to determine whether some morphological information on the biological sample, such as positions, dimensions and shapes, can be estimated. In the following, we will consequently focus on the complex scattering potential of the reconstructed objects and only plot its modulus which appears less disturbed than its real or imaginary parts [20].

Using the notations introduced in figure 2, for a monochromatic incident plane wave $U_{i n c}(\vec{r})=e^{i \overrightarrow{k_{0}}} \cdot \vec{r}$ of wave vector $\overrightarrow{k_{0}}=\frac{2 \pi n_{0}}{\lambda}\left(p_{0}, q_{0}, m_{0}\right)$, equation (3) can be rewritten to obtain the Fourier diffraction theorem [12] which links the 3D Fourier transform of the scattering potential $f$ with the 2D Fourier transform of the diffracted field $U_{d i f}$ on a given plane at $z=z^{+}$:

$$
\hat{f}(\alpha, \beta, \gamma)=\frac{4 \pi}{i k_{0}^{2}} w e^{-2 i \pi w z^{+}} \hat{U}_{d i f}\left(u, v ; z^{+}\right)
$$

where $(u, v)$ and $(\alpha, \beta, \gamma)$ are respectively the coordinates in the Fourier space on the plane $z=z^{+}$and in the Fourier space of the object, linked by the following relationships:

$$
\left\{\begin{array}{l}
\alpha=u-u_{0} \\
\beta=v-v_{0} \\
\gamma=w-w_{0}
\end{array} \text { and } w(u, v)=\sqrt{\frac{n_{0}^{2}}{\lambda^{2}}-u^{2}-v^{2}}\right.
$$

with $\left(u_{0}, v_{0}, w_{0}\right)=\frac{n_{0}}{\lambda}\left(p_{0}, q_{0}, m_{0}\right)$.

The Fourier transform ${ }^{4}$ and its inverse transform are here defined for a given function $g$ as:

$$
\begin{gathered}
\mathcal{F}(g)(u)=\hat{g}(u)=\int_{-\infty}^{\infty} g(x) e^{-2 i \pi u x} d x \\
\mathcal{F}^{-1}(\hat{g})(x)=\int_{-\infty}^{\infty} \hat{g}(u) e^{2 i \pi x u} d u
\end{gathered}
$$

Equation (5) implies that the two-dimensional surface $\hat{U}_{d i f}$ is mapped on a spherical cap (the so-called Ewald's sphere) into the 3D Fourier transform $\hat{f}$. The cap properties depend on $\overrightarrow{k_{0}}$ which gives the cap orientation via its direction $\left(p_{0}, q_{0}, m_{0}\right)$ and the cap radius via its wavelength $\lambda$.

Note here that this theorem can be used both in simulation purpose (going clockwise in figure 2 from a 3D simulated object to the diffracted waves $U_{d i f}^{j}$ in terms of lighting positions) or for

\footnotetext{
${ }^{4}$ This definition extends naturally to higher dimensions.
}

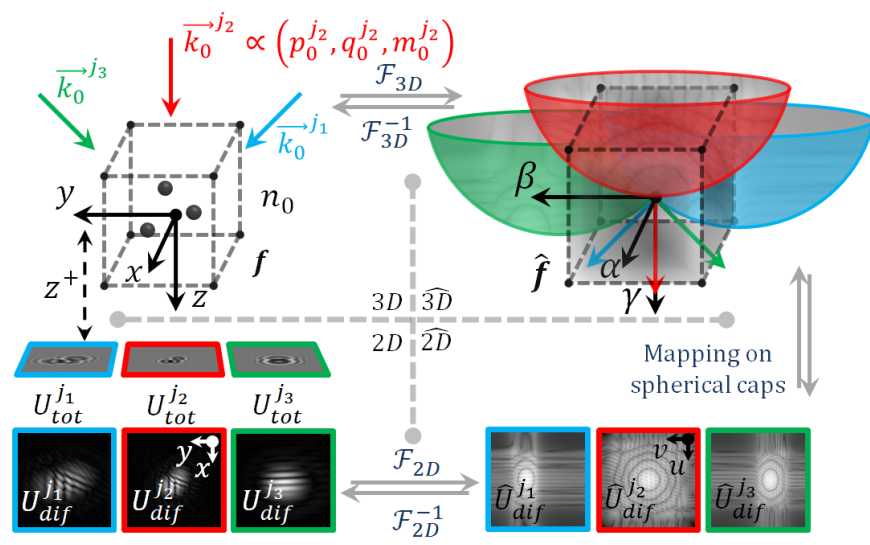

Fig. 2. Illustration of the geometrical interpretation of the Fourier diffraction theorem. A 3D Fourier transform links the 3D spatial and frequency domains of the scattering potential $f$. A 2D Fourier transform links the 2D spatial and frequency domains of the diffracted wave $U_{d i f}$ for each lighting situation $j$. A mapping on spherical caps links the 2D frequency domain of the diffracted wave and the 3D frequency domain of the object. The orientation and radius of these caps directly depend on the illumination directions $\overrightarrow{k_{0}}{ }^{j} \propto\left(p_{0}^{j}, q_{0}^{j}, m_{0}^{j}\right)$ and the associated wavelengths $\lambda^{j}$.

direct reconstruction (going counter-clockwise in figure 2 from the diffracted waves recorded by the sensor toward the retrieved object via a mapping of the Fourier domain on spherical caps).

Importantly, this theorem requires the knowledge of the diffracted wave $U_{\text {dif }}$ both in amplitude and phase, whereas as mentioned above, only $I_{t o t}=\left|U_{t o t}\right|^{2}$ is recorded by the sensor with our device.

Consequently, the inverse problem consisting in retrieving $f$ from the indirectly recorded diffracted wave $U_{d i f}$ is ill-posed due to this lack of phase information in the measurement of $U_{\text {tot }}$.

\section{D reconstruction methods}

Reconstruction methods based on the Fourier diffraction theorem (4) must deal with two inherent limitations of our in-line holographic device: the lack of phase information on the wavefront in the sensor plane, and the limited angular coverage of the acquisitions.

As mentioned earlier, the first limitation is a consequence of the fact that the sensor only records the wavefront intensity, and its phase information is lost. This lack of information leads to the well-known effect of twin-image in standard 2D lensfree microscopy and such artefact can be awaited in our 3D reconstructions if no phase information is added in the sensor plane.

The second limitation comes from the limited possibilities to turn around the object, the angular coverage being limited by the wall of the container and the fact that the sensor must stay parallel to this container to be as close as possible to the biological sample. Moreover, only a discrete and finite number $N$ of angles are acquired. This means that only a part of the Fourier space of the 3D object is accessible from a given dataset: the coefficients lying on the spherical caps corresponding to each illumination $\vec{k}_{0} j, j \in \llbracket 1, N \rrbracket$ which depends on its direction ${\overrightarrow{k_{0}}}^{j} \propto\left(p_{0}^{j}, q_{0}^{j}, m_{0}^{j}\right)$ and its wavelength $\lambda^{j}$.

To overcome these limitations, we developed three different 
but complementary methods. The first step of each method is a registration of the data. Indeed, as the illumination and the sensor turn around the sample, the hologram of a given object rotates and falls on different positions on the sensor. The use of the whole $3840 \times 2748$ pixels frame in the reconstruction method is time and memory consuming, and one can use a region of interest surrounding these holograms, as presented in [11].

Methods C.1 and C.2 are based on the Fourier diffraction theorem used to map the Fourier domain $\hat{f}$ of the 3D object $f$. Each acquisition with a different illumination gives information on coefficients of $\hat{f}$ lying on spherical caps (figure 2 used counter-clockwise). Both of these methods needs an estimation of the diffracted wave $U_{d i f}$. Method C.3 uses the Fourier diffraction theorem as a direct model for simulating the data, i.e. the recorded intensity of the total wave $U_{\text {tot }}$ (figure 2 used clockwise).

All the recorded intensities of the total transmitted waves $I_{\text {tot }}=\left|U_{t o t}\right|^{2}$ are normalised so that their background value equals to 1 , corresponding to the unperturbed theoretical incident wave $U_{i n c}(\vec{r})=e^{i \overrightarrow{k_{0}}} \cdot \vec{r}$.

\section{Phase ramp}

In this method first introduced in [11], the unknown phase on the sensor is considered to be a phase ramp, whose characteristics match the ones of the tilted illumination. To do so, the square root of $I_{t o t}$ is multiplied by a phase $\operatorname{ramp} \exp \left(i \overrightarrow{k_{0}} \cdot \vec{r}\right)$ to take into account the non-uniform phase shift induced by the tilted incidence of $\overrightarrow{k_{0}}$ relative to the sensor plane. Then we subtract the theoretical incident plane wave $U_{i n c}(\vec{r})=e^{i \overrightarrow{k_{0}} \cdot \vec{r}}$ :

$$
U_{d i f} \simeq \sqrt{I_{t o t}} \exp \left(i \overrightarrow{k_{0}} \cdot \vec{r}\right)-U_{i n c}
$$

This approximation is a needed step in the presented method, as the phase contains information about the inclination of the wavefront. Without this information, the mapping of the Fourier space with equation (12) cannot work because the computed 2D spectra will be shifted in the Fourier space. Equation (8) reintroduces the information of the illumination angle in the data. Nevertheless, it does not totally compensate the lack of phase information in the sensor plane since the phase distortion introduced by the object is not taken into account [11].

Once an approximation of $U_{\text {dif }}$ is known, it is mapped into the Fourier space of $f$ according to the Fourier diffraction theorem. To do so, equation (4) is first rewritten in terms of $(\alpha, \beta, \gamma)$ to homogenise the coordinates in the frequency domain:

$$
\hat{f}(\alpha, \beta, \gamma)=\frac{4 \pi}{i k_{0}^{2}} w^{\prime} e^{-2 i \pi w^{\prime} z^{+}} \hat{U}_{d i f}\left(\alpha+u_{0}, \beta+v_{0} ; z^{+}\right)
$$

with $\gamma=w^{\prime}-w_{0}$ and $w^{\prime}(\alpha, \beta)=\sqrt{\frac{n_{0}^{2}}{\lambda^{2}}-\left(\alpha+u_{0}\right)^{2}-\left(\beta+v_{0}\right)^{2}}$.

Equations (6) and (9) give:

$$
\hat{f}(\alpha, \beta, \gamma)=\frac{4 \pi}{i k_{0}^{2}} w^{\prime} e^{-2 i \pi w^{\prime} z^{+}} \mathcal{F}_{2 D}\left(U_{d i f} \cdot e^{-2 \pi i\left(u_{0} x+v_{0} y\right)}\right)
$$

Note that this equation is given in terms of the absolute position $(x, y)$ in the sensor plane. But, as previously mentioned, the holograms are aligned prior to the reconstruction step. $U_{d i f}$ is consequently expressed in terms of $\left(x+x_{0}, y+y_{0}\right)$, where $\left(x_{0}, y_{0}\right)=z_{s}\left(p_{0}, q_{0}\right)$ is the theoretical position of the hologram in the sensor plane at a distance $z^{+}=z_{s}$ (figure 2). Taking into account this shift in the spatial domain and according to equation (7), one finally gets:

$$
\begin{aligned}
\hat{f}(\alpha, \beta, \gamma)= & e^{-2 \pi i\left(x_{0} \alpha+y_{0} \beta\right)} \frac{4 \pi}{i k_{0}^{2}} w^{\prime} e^{-2 i \pi w^{\prime} z_{s}} \ldots \\
& \mathcal{F}_{2 D}\left(U_{d i f} \cdot e^{-2 \pi i\left(u_{0}\left(x+x_{0}\right)+v_{0}\left(y+y_{0}\right)\right)}\right)
\end{aligned}
$$

This Fourier mapping operation is performed for each $U_{d i f}^{j}$ recorded for each lighting situation ${\overrightarrow{k_{0}}}_{j}, j \in \llbracket 1, N \rrbracket$. An approximation of $f$ can then be retrieved with an inverse Fourier transform of the mapped $\hat{f}$ :

$$
\begin{aligned}
f= & \mathcal{F}_{3 D}^{-1}\left[\operatorname { m a p } \left(e^{-2 \pi i\left(x_{0}^{j} u+y_{0}^{j} v\right)} \frac{4 \pi}{i k_{0}^{2}} w^{j^{\prime}} e^{-2 i \pi w^{j \prime} z_{s}} \ldots\right.\right. \\
& \left.\left.\mathcal{F}_{2 D}\left(U_{d i f}^{j} \cdot e^{-2 \pi i\left(u_{0}^{j}\left(x+x_{0}^{j}\right)+v_{0}^{j}\left(y+y_{0}^{j}\right)\right)}\right)\right)\left.\right|_{\left\{\vec{k}_{0}^{j} \mid j=1 \rightarrow N\right\}}\right]
\end{aligned}
$$

where map (.) $\left.\right|_{\left\{\overrightarrow{k_{0}^{j}} \mid j=1 \rightarrow N\right\}}$ stands for the mapper ${ }^{5}$ of the spherical caps into the Cartesian mesh of $\hat{f}$. To avoid aliasing, both $U_{d i f}^{j}$ and $f$ are zero-padded.

This method has the advantage to reconstruct large volumes in a small amount of time. Nevertheless, it is based on a strong approximation on the phase and only a small part of the Fourier domain of the object is constrained: the coefficients on which lie the spherical caps. Hence, one can expect strong artefacts as discussed in [11].

\section{$2 D$ phase retrieval}

As presented in [10], this method aims to find a better estimate of the unknown phase in the sensor plane by performing an iterative phase retrieval. To this end, for each $2 \mathrm{D}$ picture $I_{\text {tot }}^{j}$ of the dataset $j \in \llbracket 1, N \rrbracket$, the $3 \mathrm{D}$ object to retrieve is approximated by an average median plane $t_{2 D}^{j}$. This mathematical artifice is used in a phase retrieval algorithm to get the phase at the sensor plane for this given illumination $j$. Contrary to the method exposed in [10], our phase retrieval technique is based on an inverse problem approach.

Using the notations of figure 2 , the total wave $U_{\text {tot }}$ at $z=z^{+}$ diffracted by a $2 \mathrm{D}$ complex transmissive plane $t_{2 D}$ at $z=0$ is directly given by the Rayleigh-Sommerfeld diffraction formula [21]:

$$
U_{t o t}(\vec{r})=\frac{z^{+}}{i \lambda} \iint_{\overrightarrow{r_{\mathcal{O}}} \in \mathcal{O}} t_{2 D}\left(\overrightarrow{r_{\mathcal{O}}}\right) U_{i n c}\left(\overrightarrow{r_{\mathcal{O}}}\right) \frac{e^{i k_{0}\left\|\vec{r}-\overrightarrow{r_{\mathcal{O}}}\right\|}}{\left\|\vec{r}-\overrightarrow{r_{\mathcal{O}}}\right\|^{2}} d^{2} \overrightarrow{r_{\mathcal{O}}}
$$

For low scattering objects, one can rewrite: $t_{2 D}=1+\delta t$. Equation (13) becomes:

$$
\begin{aligned}
U_{t o t}(\vec{r}) & =U_{i n c}(\vec{r})+U_{\text {dif }}(\vec{r}) \\
& =U_{i n c}(\vec{r})+\left(U_{i n c} . \delta t\right) \star\left(\frac{z^{+}}{i \lambda} \frac{e^{i k_{0} r}}{r^{2}}\right)
\end{aligned}
$$

The convolution $\star$ is computed in the padded Fourier domain. To take into account the limited coherence length $l_{c o h}$ of

\footnotetext{
${ }^{5}$ Different techniques were tested to map the discrete cap into the discrete cubic mesh: the nearest neighbour techniques and linear interpolation. As there was no noticeable differences in the results, for ease of use and rapidity, we kept a mapping of the nearest neighbour Fourier coefficients.
} 
the illumination and to reduce aliasing effects due to the high frequencies of the convolution kernel, we also added a pyramidal mask in the kernel:

$$
M_{l_{c o h}}(x, y)=\left\{\begin{array}{c}
\frac{l_{c o h}-r_{2 D}}{l_{c o h}} \text { if } r_{2 D}<l_{c o h} \\
0 \text { if } r_{2 D}>l_{c o h}
\end{array}, r_{2 D}=\sqrt{x^{2}+y^{2}}\right.
$$

Then for any $\delta t$, it exists a direct model giving the complex diffracted wave $U_{\text {dif }}(\delta t)$ at the sensor plane:

$$
U_{d i f}(\delta t)=\left(\delta t . U_{i n c}\right) \star\left(M_{l_{c o h}} \frac{z^{+}}{i \lambda} \frac{e^{i k_{0} r}}{r^{2}}\right)
$$

The inverse problem of retrieving $\delta t$ from the measurements $I_{\text {tot }}$ is ill-posed due, among others, to the lack of phase measurement on the detector plane.

We choose to resolve the reconstruction problem by minimising the following data-fidelity term:

$$
\tilde{\delta} t=\underset{\delta t}{\operatorname{argmin}}\left\|I_{t o t}-\left|U_{i n c}+U_{d i f}(\delta t)\right|^{2}\right\|^{2}
$$

The initial parameters of the experiment (here $\delta t$ ) are retrieved from the knowledge of the experimental data (here $I_{t o t}$ ) and the direct model allowing to simulate numerical data for a given set of parameters. This inverse approach allows us to model the non-linear direct process of image formation without requiring an inversion formula.

Nonetheless, minimising directly equation (17) will not be sufficient to retrieve the phase of $U_{d i f}$ since an infinite number of phase can match the recorded intensities $I_{t o t}$. One needs to add some constraints and regularisations to the minimisation problem (17). The inverse approach allows us to perform this by minimising the following criterion:

$$
\tilde{\delta t}=\underset{C(\delta t)}{\operatorname{argmin}}\left\|I_{t o t}-\left|U_{t o t}(\delta t)\right|^{2}\right\|^{2}+\mu_{L_{1}}\|\delta t\|_{L_{1}, \epsilon}+\mu_{\nabla}\|\nabla \delta t\|_{L_{1}, \epsilon}
$$

$C(\delta t)$ stands for the constraint given on the domain of admissible solutions. The central term is a sparsity prior given by:

$$
\|\delta t\|_{L_{1}, \epsilon}=\frac{1}{n b_{x} n b_{y}} \sum_{k, l} \sqrt{\left|\delta t_{k, l}\right|^{2}+\epsilon^{2}}
$$

where the indices $(k, l)$ stand for the $n b_{x} \times n b_{y}$ pixels' locations on the image grid respectively on the $x$ and $y$-axes. $\epsilon$ is a small number to ensure the differentiability of the $L_{1}$-norm in the vicinity of 0 . If one works with overall sparse objects, this aims at enforcing the fact that the expected reconstructed plane is mainly composed of isolated "particles" [22, 23].

Another a priori hypothesis is that the expected "particles" have almost uniform values inside their support. As a result, the gradient of $\delta t$ has to be almost sparse. Another interpretation is that the reconstructed image has to be smooth while preserving sharp edges at the locations of the particles. This gives the right-hand term of equation (18) which takes the form of an edge-preserving regularisation $[24,25]$. The gradient of $\delta t$ is computed as follows:

$$
\begin{aligned}
\left|\nabla \delta t_{k, l}\right|^{2}= & \frac{1}{2}\left[\left|\delta t_{k+1, l}-\delta t_{k, l}\right|^{2}+\left|\delta t_{k, l+1}-\delta t_{k, l}\right|^{2}+\cdots\right. \\
& \left.\left|\delta t_{k+1, l+1}-\delta t_{k, l+1}\right|^{2}+\left|\delta t_{k+1, l+1}-\delta t_{k+1, l}\right|^{2}\right]
\end{aligned}
$$

The parameter $\epsilon$ is again used to avoid the singularity when $\nabla \delta t=0$. It has also the property of relaxing the strong constraint put by the $L_{1}$-norm on the sharpness of edges by acting like a threshold on the gradient. Below this threshold, regularisation smooths the solution (behaving like a $L_{2}$-norm) while above this threshold, the contrast is preserved (behaving like a $L_{1}$-norm).

In order to tune an appropriate trade-off between data-fidelity and a priori information, the regularisation terms are respectively weighted by two hyper-parameters $\mu_{L_{1}}$ and $\mu_{\nabla}$.

The reconstruction consists in minimising the criterion (18), which is performed by the VMLM-B algorithm [26], a modified limited memory quasi-Newton convex optimisation method with BFGS updates and bound constraints.

Once $\delta t$ is estimated for a given illumination $j$, the corresponding diffracted wave $U_{d i f}^{j}$ can be computed via the righthand term in equation (14). When the 2D phase information of all the different acquisitions is retrieved, a 3D Fourier mapping is performed as previously exposed in the former method C.1 via equation (12) to obtain the final fully 3D reconstructed volume.

This method C.2 solves one pitfall of the previous method C.1: the phase information introduced in the reconstruction is more realistic and should reduce some artefacts. Nevertheless, it does not solve the problem of the Fourier mapping limitations: only the same coefficients on the spherical caps are accessible.

\section{D inverse problem approach}

In this last method, the Fourier diffraction theorem (6) is used as a simulation tool for a direct model (see figure 2) to implement an inverse problem approach to iteratively retrieve the $3 \mathrm{D}$ object $f$. Working directly on the full volume $f$ theoretically allows to retrieve more Fourier coefficients than methods C.1 and C.2 via and extrapolation, thanks to a priori information, of the missing frequencies which lie outside spherical caps covered by the dataset. Such an approach also allows to appropriately deal with the lack of phase information to reduce artefacts.

Using the mathematics introduced in section C.1, one gets a direct model to compute the diffracted wave $U_{\text {dif }}$ centred on its theoretical position $\left(x_{0}, y_{0}\right)$ from any scattering potential $f$ and illumination $\overrightarrow{k_{0}}$ from equations (11) and (12):

$$
\begin{array}{r}
U_{d i f}(f)=e^{2 \pi i\left(u_{0}\left(x+x_{0}\right)+v_{0}\left(y+y_{0}\right)\right)} \mathcal{F}_{2 D}^{-1}\left[e^{2 \pi i\left(x_{0} u+y_{0} v\right)} \ldots\right. \\
\left.\left.\left.\frac{i k_{0}^{2}}{4 \pi w^{\prime}} e^{2 i \pi w^{\prime} z_{s}} \operatorname{map}^{-1}\left(\mathcal{F}_{3 D}(f)\right)\right|_{k_{0}}\right)\right]
\end{array}
$$

Generalising the definitions introduced in the previous method C.2 to the third dimension, this direct model is used to define a new cost function and a new minimisation problem:

$$
\begin{array}{r}
\tilde{f}=\underset{C(f)}{\operatorname{argmin}}\left[\frac{1}{N} \sum_{j=1}^{N}\left\|I_{\text {tot }}^{j}-\left|U_{i n c}^{j}+U_{d i f}^{j}(f)\right|^{2}\right\|^{2}+\cdots\right. \\
\left.\mu_{L_{1}}\|f\|_{L_{1}, \epsilon}+\mu_{\nabla}\|\nabla f\|_{L_{1}, \epsilon}\right]
\end{array}
$$

where $C(f)$ stands for the domain constraints of the solution $\tilde{f}$.

The resolution of the minimisation problem (22) is performed with the same convex optimisation algorithm [26] introduced in method C.2.

Besides addressing the lacking phase and estimating the global Fourier transform of $f$, this method also allows to improve the data alignment. Indeed, after having performed a 
first estimation of $f$, one can use the direct model (21) to simulate a numerical dataset $\tilde{I}_{\text {tot }}^{j}=\left|U_{i n c}^{j}+U_{\text {dif }}^{j}(f)\right|^{2}, j \in \llbracket 1, N \rrbracket$. By construction, this set of simulated intensities $\tilde{I}_{\text {tot }}^{j}$ is aligned with the numerical 3D object $f$. It can consequently be used as a reference to refine the registration of the experimental data $I_{t o t}^{j}$. As presented in [11], the experimental data are aligned with the simulated intensities via a Least Squares Minimisation of the difference between the simulated intensities and the interpolation of the experimental data for a given shift.

Performing anew a 3D reconstruction of this dataset via equation (22) will increase the quality of the retrieved object. One can iterate these steps alternating between $3 \mathrm{D}$ reconstruction and data alignment. This refinement cannot be performed with the first two methods.

\section{D cell cultures}

The different 3D reconstruction approaches were tested and compared on two types of cell models which lead to different morphologies when grown in 3D scaffolds.

\section{Human Umbilical Vein Endothelial Cells (HUVEC)}

HUVEC cells are primary cells extracted from human neonatal umbilical cords used for many vascular biology research applications, such as inflammation, angiogenesis and blood clotting. The cells were purchased from Millipore ${ }^{\mathrm{TM}}$. The culture was maintained in a sub-confluent state in culture Petri Dish $\left(113 \mathrm{~cm}^{2}\right)$ coated with fibronectin at $37^{\circ} \mathrm{C}$. HUVEC cells were grown in EndoGro basal medium (Millipore ${ }^{\mathrm{TM}}$ ) supplemented with $5 \mathrm{ng} / \mathrm{mL}$ of rhVEGF, rhEGF, rhFGFb respectively, $15 \mathrm{ng} / \mathrm{mL}$ rh IGF-1, $10 \mathrm{mM}$ L-glutamine, $0.75 \mathrm{U} / \mathrm{mL}$ heparin sulfate, $1 \mu \mathrm{g} / \mathrm{mL}$ hyroscortisone hemisucinate, $50 \mu \mathrm{g} / \mathrm{mL}$ ascorbic acid, and 10\% FBS.

For the final cell culture, the cells were grown in Matrigel ${ }^{\circledR}$ (BD Biosciences) according to a no top-coat protocol. For polymerisation, Matrigel ${ }^{\circledR}$ was incubated for 30 minutes at $37^{\circ} \mathrm{C}$ and 4000 to 10000 cells were seeded and allowed to adhere for approximately 45 minutes. Then their complete medium was slowly poured over the attached cells.

All cells were routinely cultured in a humidified atmosphere with $5 \% \mathrm{CO}_{2}$ at $37^{\circ} \mathrm{C}$ for a day.

\section{Prostate Epithelial Cells (RWPE1)}

RWPE1 cell line was obtained from ATCC. This cell line was derived from non-neoplastic human prostate epithelial cells by immortalisation with human papillomavirus. RWPE1 cells were used as a model for normal prostate epithelial cell behaviour as characterised by a polarised acinar morphology in 3D cultures. RWPE1 cells were maintained in KSFM (Life Technologies) supplemented with $5 \mathrm{ng} / \mathrm{mL}$ Epidermal Growth Factor (Life Technologies), $50 \mu \mathrm{g} / \mathrm{mL}$ Bovine Pituitary Extract (Life Technologies) and 1\% Penicillin-Streptomycin (Life Technologies). Cells were passaged upon $70 \%$ confluence and seeded at 20000 cells $/ m L$ density. All cells were routinely cultured in a humidified atmosphere with $5 \% \mathrm{CO}_{2}$ at $37^{\circ} \mathrm{C}$.

Cell number and viability was measured by trypan blue dye exclusion staining using an EVE ${ }^{\mathrm{TM}}$ Automatic Cell Counter (NanoEnTek).

For the final 3D cell culture, Matrige ${ }^{\circledR}$ (BD Biosciences) microbeads were produced using a microfluidic chip with flow focusing geometry as previously described in [27]. For the acinar morphogenesis assay, RWPE1 cells in Matrigel ${ }^{\circledR}$ microbeads were allowed to develop into organoids for 7 days in KSFM (Life
Technologies) supplemented with $50 \mathrm{ng} / \mathrm{mL}$ Epidermal Growth Factor (Life Technologies), 2\% Foetal Bovine Serum (Life Technologies), $1 \%$ Matrige ${ }^{\circledR}$ and $1 \%$ Penicillin-Streptomycin (Life Technologies) in multiwell plates. Cell culture medium was changed every two days.

Finally, capsules were washed twice with medium and were then embedded in Matrigel ${ }^{\circledR}$ deposited at the center of Greiner petri dishes (Sigma-Aldrich) and allowed to polymerise for 30 minutes at $37^{\circ} \mathrm{C}$. Culture medium was subsequently added.

\section{Acquisitions of the microscope views (RWPE1)}

For comparison purpose, microscope acquisitions were performed on the RWPE1 cell culture. The organoids in capsules were labelled with $10 \mu \mathrm{M}$ CellTracker ${ }^{\mathrm{TM}}$ Orange CMTMR dye (Thermo Fisher Scientific) in media for 30 minutes at $37^{\circ} \mathrm{C}$. Finally, the RWPE1 culture was fixed using $2 \%$ paraformaldehyde $1 \%$ glutaraldehyde for $20 \mathrm{~min}$.

During these steps, the cell culture could have been slightly modified and some structures washed away. As the aim was to compare the $z$-positioning and the shape of some objects, it was however deemed sufficient.

$z$-stacks of phase and fluorescent images were acquired using an AxioObserver.Z1 inverted microscope (Zeiss) with a NAchroplan 5x/0.13 Ph0 air objective mounted with an AxioCam 503 monochrome digital camera. Acquisitions were performed on 150 slices equally spread on $\Delta z=400 \mu \mathrm{m}$.

\section{RESULTS}

\section{Comparison of the three reconstruction methods Reconstruction parameters}

The two 3D cell cultures were similarly processed.

Acquisitions Both datasets were composed of $3 \times 16$ acquisitions done at 16 different angles $\varphi \in\left\{0^{\circ}, 282^{\circ}\right\}, \Delta \varphi=$ $18.8^{\circ}$ in the three available wavelengths of the $R G B$ LED $\lambda \in\{450,520,630 \mathrm{~nm}\}$. In each dataset, a region of interest of $1024 \times 1024$ pixels $\left(1.7^{2} \mathrm{~mm}^{2}\right)$ was selected. The only limitation in this selection is that the observed region must be in all the pictures of the dataset. We chose a representative region of each dataset by encompassing several typical structures, i.e. branches for the HUVEC network, and capsules and organoids for the RWPE1 culture.

Reconstruction volume To reduce the computing time and the memory consumption, the final reconstruction was composed of $512 \times 512 \times 300$ voxels of $3.34 \times 3.34 \times 5.32 \mu \mathrm{m}^{3}$ for a global volume of $1.7 \times 1.7 \times 1.6 \mathrm{~mm}^{3}=4.7 \mathrm{~mm}^{3}$. The voxels are not orthonormal to take into account the distortion on the $z$-axis induced by the refraction between the air of refractive index $n_{\text {air }}$ and the culture medium of refractive index $n_{0}$. Indeed, for an illumination tilted of an angle $\theta_{\text {air }}$ in the air, everything acts as if the sample was illuminated with an angle $\theta_{0}$ verifying : $n_{\text {air }} \cdot \sin \theta_{\text {air }}=n_{0} \cdot \sin \theta_{0}$. This leads to a scaling factor ${ }^{6}$ on the $z$-axis equal to:

$$
\frac{z_{0}}{z_{\text {air }}}=\sqrt{\frac{n_{0}^{2} / n_{\text {air }}^{2}-\sin ^{2} \theta_{\text {air }}}{1-\sin ^{2} \theta_{\text {air }}}}
$$

${ }^{6}$ In the present case, with $\theta_{\text {air }}=45^{\circ}, n_{\text {air }} \simeq 1$ and $n_{0}=1.33$ in the water, the effective angle seen by the sample is $\theta_{0} \simeq 32^{\circ}$, leading to a scaling factor $z_{0} / z_{\text {air }} \simeq 1.59$. 
Method C.2 The three available wavelengths were used together to improve the $2 \mathrm{D}$ phase retrieval via the minimisation problem (18). As mentioned earlier for the 3D scattering potential, the effective 2D transmissive plane $\delta t$ is supposed to be independent of the illumination wavelength. To do so, the data fidelity term in equation (18) was simply modified to be the sum of the $n_{\lambda}=3$ data fidelity terms at each wavelength:

$$
\left\|I_{t o t}-\left|U_{t o t}(\delta t)\right|^{2}\right\|^{2} \leftrightarrow \frac{1}{n_{\lambda}} \sum_{\lambda_{j}}\left\|I_{\text {tot }}^{\lambda_{j}}-\left|U_{\text {tot }}^{\lambda_{j}}(\delta t)\right|^{2}\right\|^{2}
$$

To insure non-emissive objects, the constraint $C(\delta t)$ was chosen to force $\delta t$ to have a negative real part: $\mathcal{R}(\delta t)<0$. $\epsilon$ was set to $10^{-6}, l_{\text {coh }}$ to $500 \mu \mathrm{m}$ and the hyper-parameters to $\mu_{L_{1}}=0.5$ and $\mu_{\nabla}=0.1$. The number of iterations was fixed to 100 .

Method C.3 For the 3D inverse problem approach, the iterative process was split in seven batches of ten iterations in between which a data registration was performed. The constraints were set to $\mathcal{R}(f)>0$ and $\mathcal{I}(f)>0$ which leads for a small $\delta n=$ $n-n_{0}$ to an absorbent and dephasing object. $\epsilon$ was set to $10^{-6}$.

Something which was also tested in this work was to change the weight of the hyper-parameters along this iterative process. For the first guesses, for example, one can start with a small regularisation on sparsity and increase the hyper-parameter $\mu_{L_{1}}$ afterwards to enforce the constraint. To do so, $\mu_{L_{1}}$ was set to vary from 0 to $100([0,0,0,10,10,100,100]$ for the HUVEC dataset and $[0,0,0,0,10,10,100]$ for the RWPE1 dataset). On the other hand, especially for complex samples, one can start with a problem strongly regularised in terms of localisation with high values for $\mu_{\nabla}$ : the data fidelity is degraded, but the overregularisation forces the reconstructed object to be localised, and consequently the simulated data to be well aligned, bettering the registration step. Once the alignment of the experimental data is more accurate, the hyper-parameter $\mu_{\nabla}$ can be set to lower values, increasing the data fidelity relative weight in the cost function. $\mu_{\nabla}$ was consequently set to vary from 3 to 1 for the HUVEC dataset $([3,1,1,1,1,1,1])$ and from 5 to 1 for the RWPE1 dataset $([5,5,3,1,1,1,1])$.

\section{Performances of the different methods}

Method C.2 As expected, the iterative RGB 2D phase retrieval algorithm developed for method C.2 reduces the numerous artefacts introduced by the lack of phase information (see figure 3). The sparsity constraint efficiently cleans the noise and the twinimage signal present in the background of figure 3.b: the background on figure 3.c is homogeneous with overlying sharp and contrasting objects.

After a rapid drop in the first 20 iterations for each dataset (see figure 4), the cost function of equation (18) barely decreases after the $50^{\text {th }}$ iteration.

Method C.3 The convergence curves for the 3D inverse problem (see figure 5) are less intuitive as they are not strictly decreasing. This is due to the fact that every 10 iterations, a data alignment refinement occurs and the values of the hyper-parameters may change. Both of these effects are visible on the curves.

If the registration step does not have a visible effect on the HUVEC convergence curve, its effect is noticeable between the first and the second batch of the RWPE1 dataset reconstruction. Indeed, between these two batches, at the $10^{\text {th }}$ iteration, the value of the hyper-parameters is unchanged $\left(\mu_{L_{1}}=0\right.$ and $\left.\mu_{\nabla}=5\right)$ and so is the value of the last two terms of the cost function (22). The change in the cost function from more than $16 \%$ to

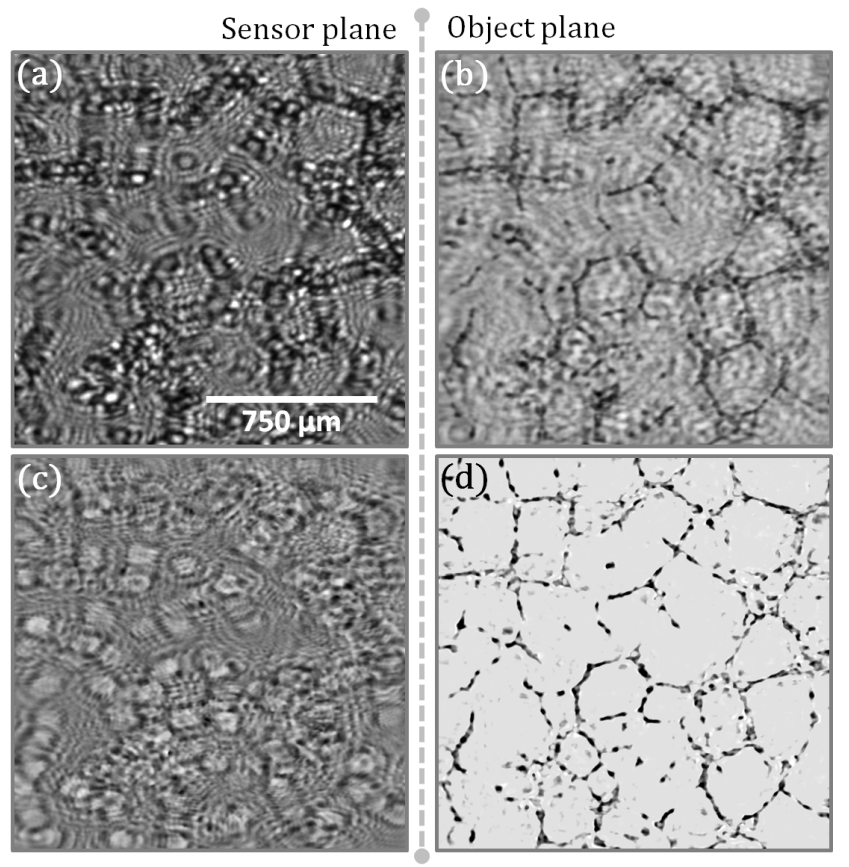

Fig. 3. Example of a $R G B$ phase retrieval performed on the first illumination position in the HUVEC dataset. (a) Raw acquisition by the sensor with the green illumination. (b) Modulus of a simple 2D back-propagation of the green intensity to the object plane. The classical twin-image effect is clearly visible around the isolated cells and the branches of the HUVEC network. (c) Recovered phase information in the sensor plane for the green illumination after the iterative $R G B$ retrieval. (d) The retrieved $2 \mathrm{D}$ object modulus. Most of the artefacts are erased by the regularised inverse problem approach.

less than $14 \%$ can therefore only be attributed to the diminution of the data fidelity term because of a better agreement between the experimental and simulated data.

The noticeable upward jumps are linked with the change of the value of the hyper-parameter $\mu_{L_{1}}$. It gains a factor 10 at the $30^{\text {th }}$ and the $50^{\text {th }}$ iterations for the HUVEC reconstruction and at the $40^{\text {th }}$ and the $60^{\text {th }}$ iterations for the RWPE1 reconstruction.

Reconstruction time The reconstruction time with the phase ramp method C.1 is directly the time of the Fourier mapping operation and is quite fast $(\sim$ mins) (see table 1$)$.

\begin{tabular}{|c|c|c|c|}
\cline { 2 - 4 } \multicolumn{1}{c|}{} & Method C.1 & Method C.2 & Method C.3 \\
\hline HUVEC & $\sim 1 \mathrm{~min}$ & $\sim 1 h 20 \mathrm{~min}$ & $\sim 7 h$ \\
\hline RWPE1 & $\sim 1 \mathrm{~min}$ & $\sim 2 h$ & $\sim 10 h$ \\
\hline
\end{tabular}

Table 1. Comparison of the reconstruction times with the different methods. The increase in the complexity of the reconstruction method significantly lengthens the computation time. They were obtained with our Matlab ${ }^{\circledR}$ code running on a Intel(R) Xeon(R) CPU E5-2620 v3 @ $2.40 \mathrm{GHz}$ processor. The code was not fully optimised, thus the given times must be considered as an order of magnitude for comparison purposes.

As expected, most of the reconstruction with the method C.2 is spent in the $2 \mathrm{D}$ phase retrieval: several minutes for each of the 


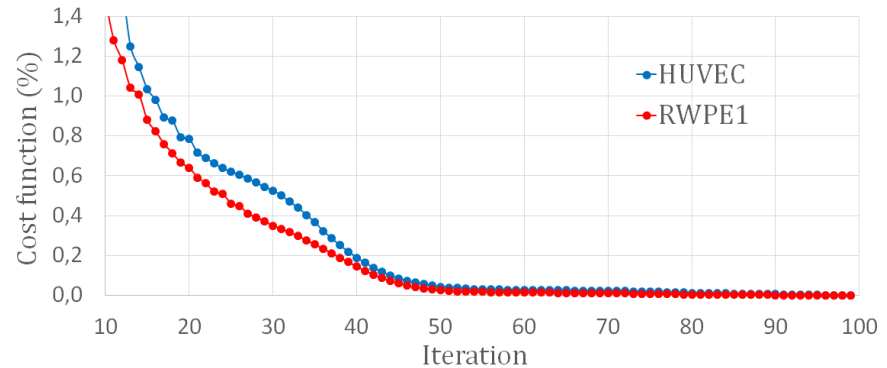

Fig. 4. Convergence curves of the RGB phase retrieval C.2 performed on the first illumination position in the HUVEC (see figure 3) and RWPE1 datasets. The curves were normalised to their values at the first and the last iterations.

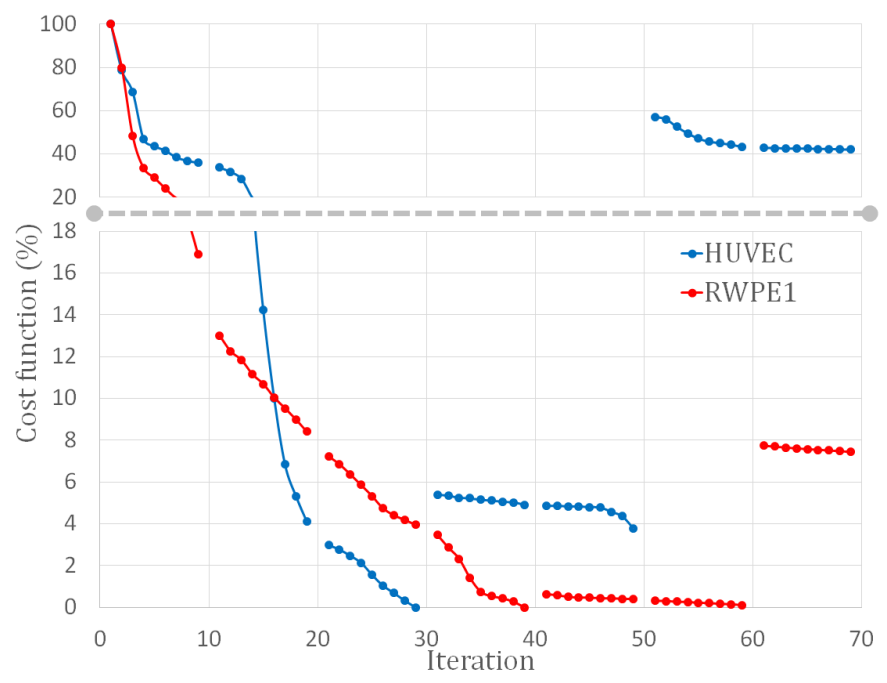

Fig. 5. Convergence curves of the iterative $3 \mathrm{D}$ inverse problem C.3 for the two datasets. The curves were normalised to their values at the first and the last iterations. For each dataset, the batches of 10 iterations are separated by a blank in the curve to emphasise the iterations where the alignment of the data is refined and where a change in the hyper-parameters can occur.

16 lighting positions. In details, the final reconstruction times for the HUVEC and RWPE1 datasets are $\sim 16 \times 5+2 \mathrm{~min}$ and $\sim 16 \times 7+2 \mathrm{~min}$. The last $2 \mathrm{~min}$ correspond to the final Fourier mapping operation.

Unsurprisingly, the $3 \mathrm{D}$ inverse problem approach C.3 is the slowest one. The running time being composed of roughly $60 \%$ for the minimisation of the problem (22) and $40 \%$ for the data registration refinement.

\section{$3 D$ reconstructions}

In this section we compare the three methods via 3D reconstructions on the two experimental datasets.

HUVEC reconstruction It clearly appears on the volumes slices (see figures 6.c-e) that the methods C.2 and C.3 strongly diminish the artefacts of the phase ramp solution C.1 by cleaning the twinimage from the background. One can nevertheless see on the orthogonal views (see figures 6.i-k) that strong artefacts remain in the reconstructions performed with the method C.2. They are due to the limited angular coverage: the cone shapes around the contrasted objects are characteristic of the angle $\theta$ at which the sample is illuminated.
For morphological and positioning analysis, method C.2 appears to be sufficient compared to method C.3 (see figures 6.d-e). The gain of contrast in the plotted profiles on figures 6.f-h does not justify the high calculation time of method C.3, since the two methods give similar results in terms of segmentation capabilities.

RWPE1 reconstruction The HUVEC network is overall planar compared to the RWPE1 cell culture which is more complex with overlapping structures spread on a large scale along the $z$-axis (see figure 7.b). It appears that the previous remarks on the artefacts reduction still holds (see figures 7.c-e. and 7.i-k).

Besides, some 3D structures only contrast from the background with method C.3. This is particularly visible on the profiles (see figures 7.c-h) in which the signal of small individual objects strongly peaks (green arrows) in all the reconstructions, whereas some organoids are only visible with the inverse problem approach C.3 (red arrows). In addition, the segmentation between the three structures between the blue brackets along the dashed-lines appears possible only with method C.3.

In order to better quantify these differences in the reconstructions quality, a contrast to noise ratio $R$ was estimated on specific regions of interest and compared for the three methods. A region of $45 \times 45$ pixels was selected in a region seemingly without any reconstructed object to compute the mean value $m_{b}$ and the standard deviation $\sigma_{b}$ of the background signal. Then spots of $3 \times 3$ pixels were selected to estimate their mean intensity $m_{s}$. The contrast to noise ratio was then computed as follows:

$$
R=\frac{m_{s}-m_{b}}{\sigma_{b}}
$$

This does not pretend to be a rigorous signal to noise ratio estimation. Indeed, we do not know the real signal to actually compare it with our reconstructions. Moreover, these ratios do not reflect the efficiency of the different methods to reduce the artefacts: the background is estimated in a region where they seem to appear minimal, whereas they can peak rather high (see the twin-image in the phase ramp reconstructions on figures 6.c and 7.c). These ratios consequently give only an idea on how the different algorithms increase the signal intensity compared to the background.

First of all, the different contrasts (see table 2) confirm that isolated objects are well reconstructed with a contrast higher than 15 compared to the background $\left(R_{0}\right.$ and $\left.R_{3}\right)$ for all the reconstruction techniques. The value $R_{1}$ confirms that some structures such as the spot 1 on figures 7.c-e are undistinguishable from the background with the 2D phase retrieval C.2 (see figure 7.d). One needs the 3D inverse problem method C.3 to extract these structures from the background intensity.

The contrast to noise ratio is higher with method C.3. One could have nevertheless expected a better contrast based on the scales of the profiles (see figures 6.f-g and 7.f-g). This comes from the standard deviation of the background which gains a factor of $\sim 12$ with this method compared to methods C.1 and C.2.

Concerning the sectioning on the $z$-direction, the $3 \mathrm{D}$ view of figure 7.b shows that objects are widely spread in the three dimensions and artefacts on this direction seem limited. Looking more closely at the orthogonal views (see figures 7.i-k), it appears once again that only the $3 \mathrm{D}$ regularised problem approach is able to clean the artefacts to correctly retrieve the objects. Indeed, one can see that the isolated and small objects that are blurred by the twin-image with the simple phase ramp method C.1 are well reconstructed by the $2 \mathrm{D}$ phase retrieval algorithm C.2 (see 

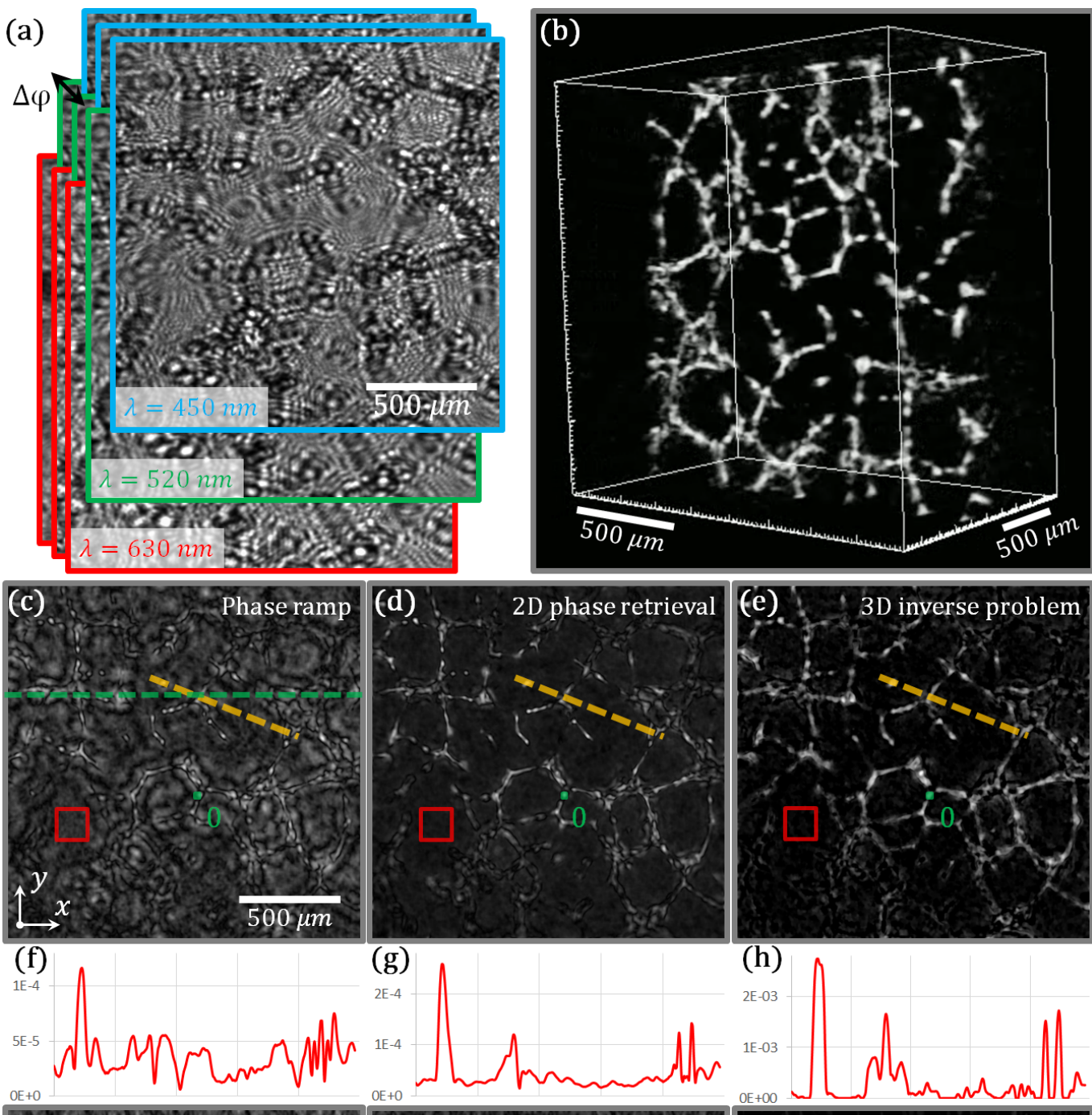

(h)
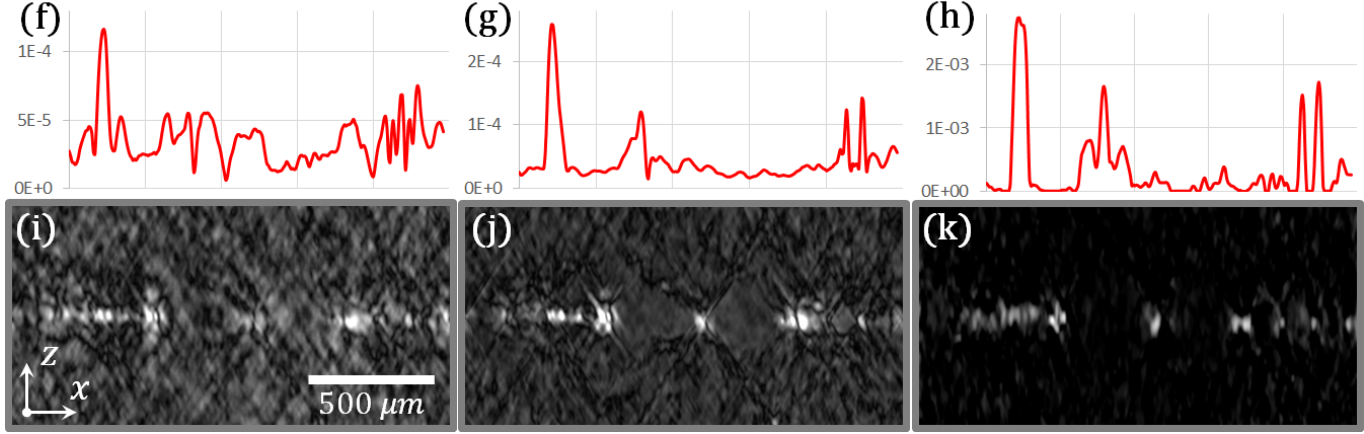

Fig. 6. Comparison of the reconstruction methods on a HUVEC network. The cells spread on the Matrigel ${ }^{\circledR}$ surface and the final network is overall planar. (a) Raw data at the three wavelengths and at different angles. (b) 3D visualisation of the reconstructed volume with the 3D inverse problem method (see visualisation 1). Reconstruction parameters: $\varphi \in\left\{0^{\circ}, 282^{\circ}\right\}, \Delta \varphi=18.8^{\circ}$, $\theta=45^{\circ}, \lambda=R G B, z_{s}=$ $3.3 \mathrm{~mm}, 512 \times 512 \times 300$ voxels of $3.34 \times 3.34 \times 5.32 \mu^{3}$. Final volume: $1.7 \times 1.7 \times 1.6 \mathrm{~mm}^{3}=$ $4.7 \mathrm{~mm}^{3}$. (c-e) Comparison of the three methods for a volume slice at $z=0 \mu \mathrm{m}$. The red square and the green spot (0) represent respectively the areas on which the background and the signal were estimated. (f-h) Profiles drawn along the yellow dashed-lines in figures 6.(c-e). (i-k) Cropped views on the $x z$ plane orthogonal to the green dashed-line.

\begin{tabular}{|c|c|c|c|c|c|c|c|c|c|c|c|c|}
\hline & \multicolumn{4}{|c|}{ HUVEC } & \multicolumn{8}{|c|}{ RWPE1 } \\
\hline & $m_{b}\left(10^{-5}\right)$ & $\sigma_{b}\left(10^{-6}\right)$ & $m_{s}\left(10^{-4}\right)$ & $R_{0}$ & $m_{b}\left(10^{-5}\right)$ & $\sigma_{b}\left(10^{-6}\right)$ & $m_{s}^{1}\left(10^{-5}\right)$ & $R_{1}$ & $m_{s}^{2}\left(10^{-5}\right)$ & $R_{2}$ & $m_{s}^{3}\left(10^{-4}\right)$ & $R_{3}$ \\
\hline Method C.1 & 2.88 & 6.16 & 1.22 & 15 & 2.51 & 5.74 & 4.44 & 3.4 & 7.68 & 9 & 1.12 & 15 \\
\hline Method C.3 & 8.35 & 79.8 & 26.6 & 32 & 5.71 & 66.9 & 71.3 & 9.8 & 13.7 & 20 & 40.1 & 59 \\
\hline
\end{tabular}

Table 2. Details of the estimation of the contrast $R$ for the different areas spotted on figures 6.c-e and 7.c-e. The exponents are coherent with the numbering of the spots on these figures.

figures 7.i and j). Added to methods C.1 and C.2, method C.3 efficiently cleans the remaining artefacts due to the lack of angular coverage. Objects in the vicinity of the organoids are now visible and well separated. Nonetheless, their shape normally spherical appears rather straight. This is once again due to the impossibility of acquiring angles widely distributed in elevation $(\theta$-angle on figure 1): the algorithm cannot recover the information which is in the zones of shadow of the organoids.

\section{Comparison with a standard microscope}

To further study the sectioning capabilities of the device and the algorithms on the $z$-axis, a comparison was made with acquisitions from a standard microscope.

To do so, a fully $3 \mathrm{D}$ reconstruction was performed with a higher resolution on the RWPE1 cell culture with the 3D inverse problem approach C.3. The region of interest is framed by a 

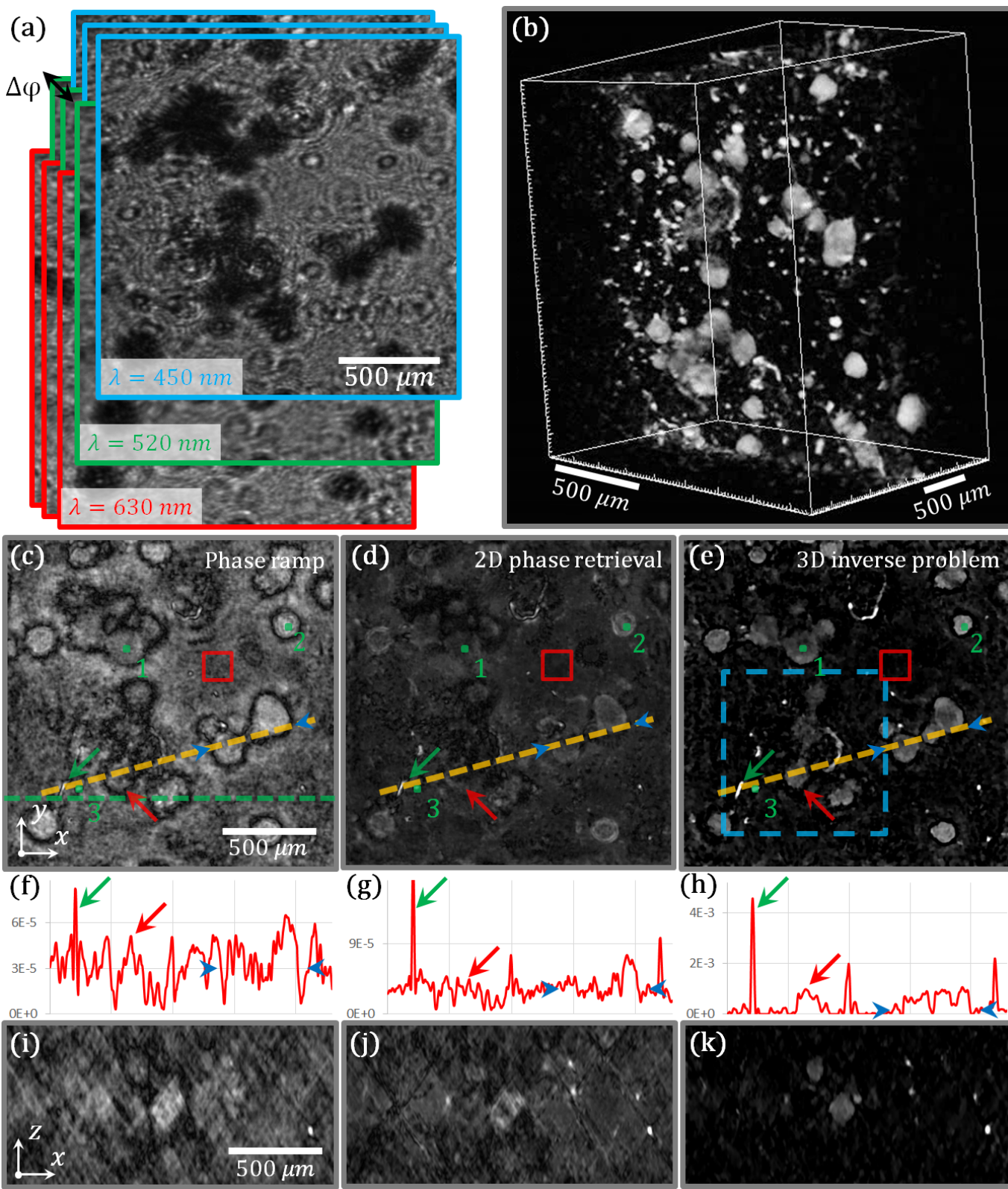

(g)
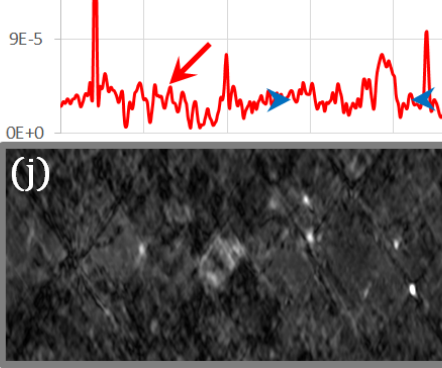

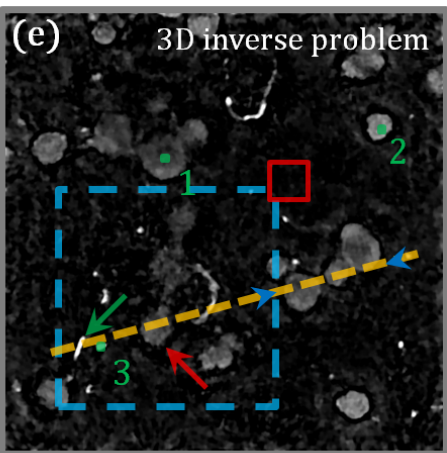

(h)
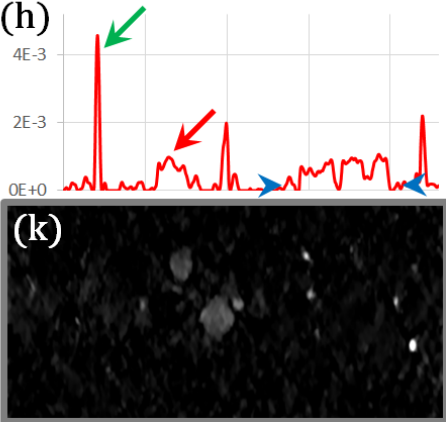

Fig. 7. Comparison of the reconstruction methods on a RWPE1 cell culture. The cells tend to form organoids when embedded in Matrigel ${ }^{\circledR}$. (a) Raw data at the three wavelengths and at different angles. (b) 3D visualisation of the reconstructed volume with the 3D inverse problem method (see visualisation 2). Reconstruction parameters: $\varphi \in\left\{0^{\circ}, 282^{\circ}\right\}, \Delta \varphi=18.8^{\circ}$, $\theta=45^{\circ}, \lambda=R G B, z_{s}=$ $3.3 \mathrm{~mm}, 512 \times 512 \times 300$ voxels of $3.34 \times 3.34 \times 5.32 \mu^{3}$. Final volume: $1.7 \times 1.7 \times 1.6 \mathrm{~mm}^{3}=$ $4.7 \mathrm{~mm}^{3}$. (c-e) Comparison of the three methods for a volume slice at $z=-48 \mu \mathrm{m}$. The red square and the green spots $(1,2,3)$ represent respectively the areas on which the background and the signal were estimated. The blue square is the zone which was reconstructed with a higher resolution for comparison with a standard microscope. (f-h) Profiles drawn along the yellow dashed-lines in figures 7.(c-e). (i-k) Cropped views on the $x z$-plane orthogonal to the green dashed-line. blue dashed square on figure 7.e. The dataset was composed of $3 \times 31$ acquisitions done at 31 different angles $\varphi \in\left\{0^{\circ}, 282^{\circ}\right\}$, $\Delta \varphi=9.4^{\circ}$ in the three available wavelengths of the RGB LED. The region of interest was composed of $512 \times 512$ pixels $(855 \times$ $\left.855 \mathrm{\mu m}^{2}\right)$. The final reconstruction was composed of $512 \times 512 \times$ 300 voxels of $1.67 \times 1.67 \times 2.66 \mu^{3}$ for a global volume of $855 \times 855 \times 800 \mu^{3}=0.584 \mathrm{~mm}^{3}$.

The iterative process was split in twenty batches of ten iterations in between which a data registration was performed for the first fourteen batches. The constraints were set to $\mathcal{R}(f)>0$ and $\mathcal{I}(f)>0$ and the hyper-parameters were varying from 0 to 1000 for $\mu_{L_{1}}$ and varying from 5 to 0.5 for $\mu_{\nabla}$.

The total reconstruction time was $\sim 30 h$ whose $\sim 12 h$ were dedicated to the data registration refinement.

The reconstruction quality is degraded on the $z$-direction on the final reconstructed volume (see figure 8). This is due to the acquisition geometry: it is impossible to rotate the sample to acquire more specific data on the $z$-direction. This loss of information leads to the limited number of coefficients acces- sible in the Fourier domain of the object as mentioned above. Nevertheless, the 3D regularisation compensates this lack of viewing angles. Even if all the information is not retrieved, this algorithm allows to clean and reduce classical artefacts which appears in classical tomographic algorithms with limited angular coverage [28].

The shape of the organoids can be reconstructed as well as some information inside the structures such as global changes of density (see figures 8.a and c)). We insist here again that this is not quantitative (see 2.B). What seems to be a bridge is visible between two planes at different $z$ (see figure 8.b).

We compared the $3 \mathrm{D}$ reconstruction with microscope views (see figure 9). The main structures are common to all the views. Nevertheless, as mentioned earlier, in the reconstructed volume, single objects (red arrows) artificially contrast more than extended structures (blue arrows).

Figure 10 presents the comparison of different slices of the fully 3D reconstructed volume with their microscope counterparts. Despite a non-quantitative contrast, one can see that the 

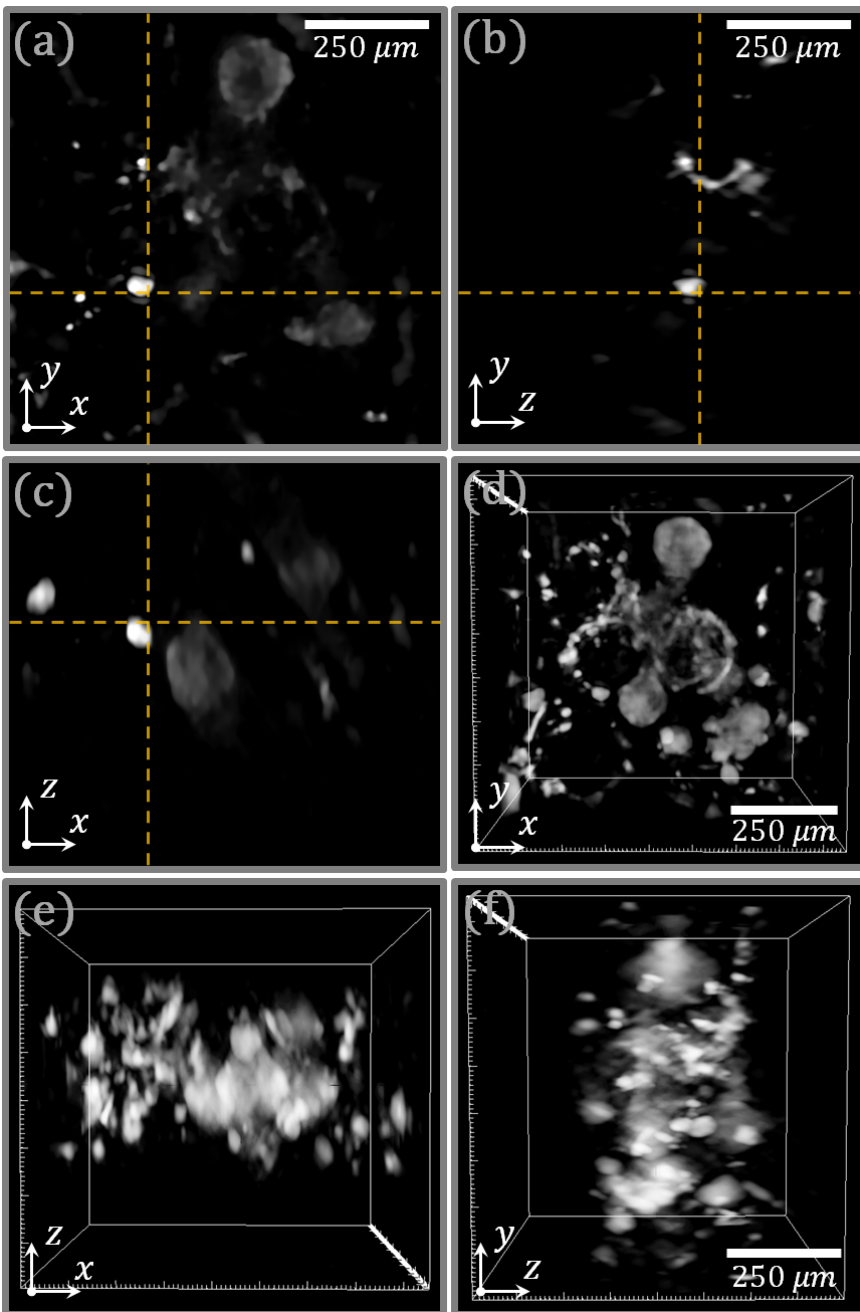

Fig. 8. Visualisations of the reconstructed volume of the RWPE1 dataset at full resolution with the $3 \mathrm{D}$ inverse problem method (see visualisation 3). Reconstruction parameters: $\varphi \in\left\{0^{\circ}, 282^{\circ}\right\}$, $\Delta \varphi=9.4^{\circ}, \theta=45^{\circ}, \lambda=R G B, z_{s}=3.52 \mathrm{~mm}, 512 \times 512 \times 300$ voxels of $1.67 \times 1.67 \times 2.66 \mu^{3}$. Final volume: $855 \times 855 \times$ $800 \mu m^{3}=0.584 \mathrm{~mm}^{3}$. (a-c) Orthogonal slices based on a $x y$ slice at $z=61 \mu \mathrm{m}$. (d-f) orthogonal 3D rendering of the fully 3D reconstructed volume. All the views are orthonormal.

main structures are accurately reconstructed both in terms of positioning and morphology.

The $z$-sectioning also appears more selective compared with the focused/unfocused criterion and is able to separate extended overlapping structures (figures 9.d-f and 10). For example, the organoid on the $z=193 \mu \mathrm{m}$ slice is well set apart from the cellular branch at $z=-33 \mu \mathrm{m}$ without any bridging artefact on the $z=105 \mu \mathrm{m}$ slice. Another example is the structure at the bottom right of the $z=-33 \mu \mathrm{m}$ slice: it appears separated from the underlying slightly unfocused small organoid visible on the microscope view. This small structure is actually reconstructed at $z=-84 \mu m$ (see visualisation 5).

In the slice $z=-126 \mu \mathrm{m}$, some structures are still visible despite the fact that this slice is the deepest one into the object and consequently beyond the limits of the model (3) since the incident wavefront crossed the entire sample.

Let's also mention here that with the fully $3 \mathrm{D}$ reconstruction, (a) Lens-free

(b) Phase contrast

(c) Fluorescence
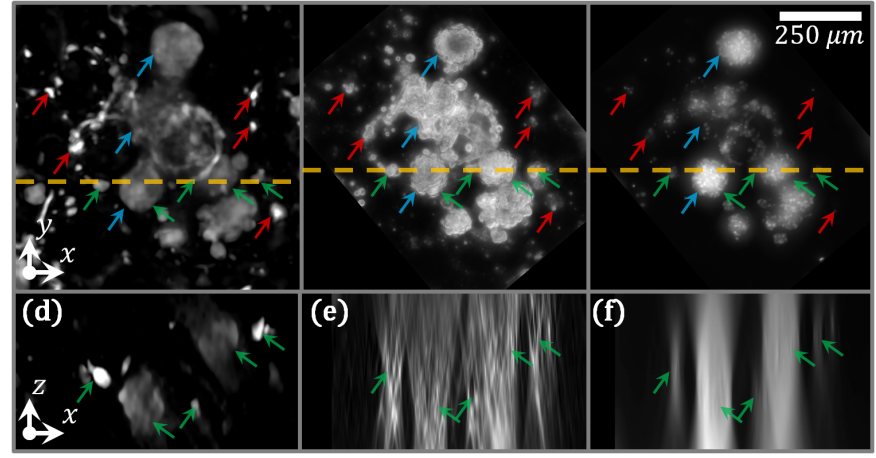

Fig. 9. Comparison between the integration of the $z$-stacks of the fully 3D reconstructed volume (a - average intensity), the phase contrast ( $b$ - average intensity) and the CellTracker ${ }^{\mathrm{TM}}$ vi- $^{-}$ sualisation (c - max intensity). (d-f) Orthogonal slices of the different volumes on the $x z$-plane along the corresponding yellow dashed-lines. The green arrows point at different objects of interest. For the phase contrast and fluorescence imaging, the arrows are placed were the objects seem at their best focus on the $x y$-plane (see visualisations 4 and 5 for more detailed views).

one can see that the two cellular branches at $z=193 \mu \mathrm{m}$ and $z=105 \mu \mathrm{m}$ are actually bridged in the third dimension: this is the branch we mentioned above on figure $8 . \mathrm{b}$ in the top left quadrant.

\section{CONCLUSION AND DISCUSSION}

We presented a novel tool to perform acquisitions on large 3D cell cultures. Based on the in-line holographic principle, it can image unlabelled and unstained living samples. To overcome the limitations raised by such a microscope, that is to say the lack of phase information on the data and the limited angular coverage, we developed three dedicated algorithms.

We showed that these algorithms are able to retrieve the 3D object but with different qualities in terms of contrast to noise ratio and computational time. Giving the result in a single pass, the algorithm based on a phase ramp is fast but leads to a signal which can be hard to distinguish from the artefacts and the noise. Providing the best contrast, the algorithm based on the 3D inverse problem approach can nevertheless be extremely time consuming.

It appears then that the choice to use either an algorithm or another will depend on the targeted application. To identify isolated single cells in a $3 \mathrm{D}$ volume, which provide a strong signal, the first algorithm can be sufficient. On the other hand if one aims at reconstructing complex overlapping structures, only the $3 \mathrm{D}$ regularised iterative reconstruction can provide a more pertinent result.

Comparison with standard microscope views showed that the fully 3D reconstructions are accurate in terms of morphology and positioning. The proposed lens-free device provides a cheap and easy to use tool with a good sectioning in the $z$-direction on large volumes. Nonetheless, it has been shown that the reconstructions are not quantitative in terms of contrast (figure 9). The small objects will appear highlighted compared to larger structures. This is a limitation of the method and makes the image prone to interpretation errors.

Beyond this proof of concept, some work remains to be done, especially in terms of computational time. 


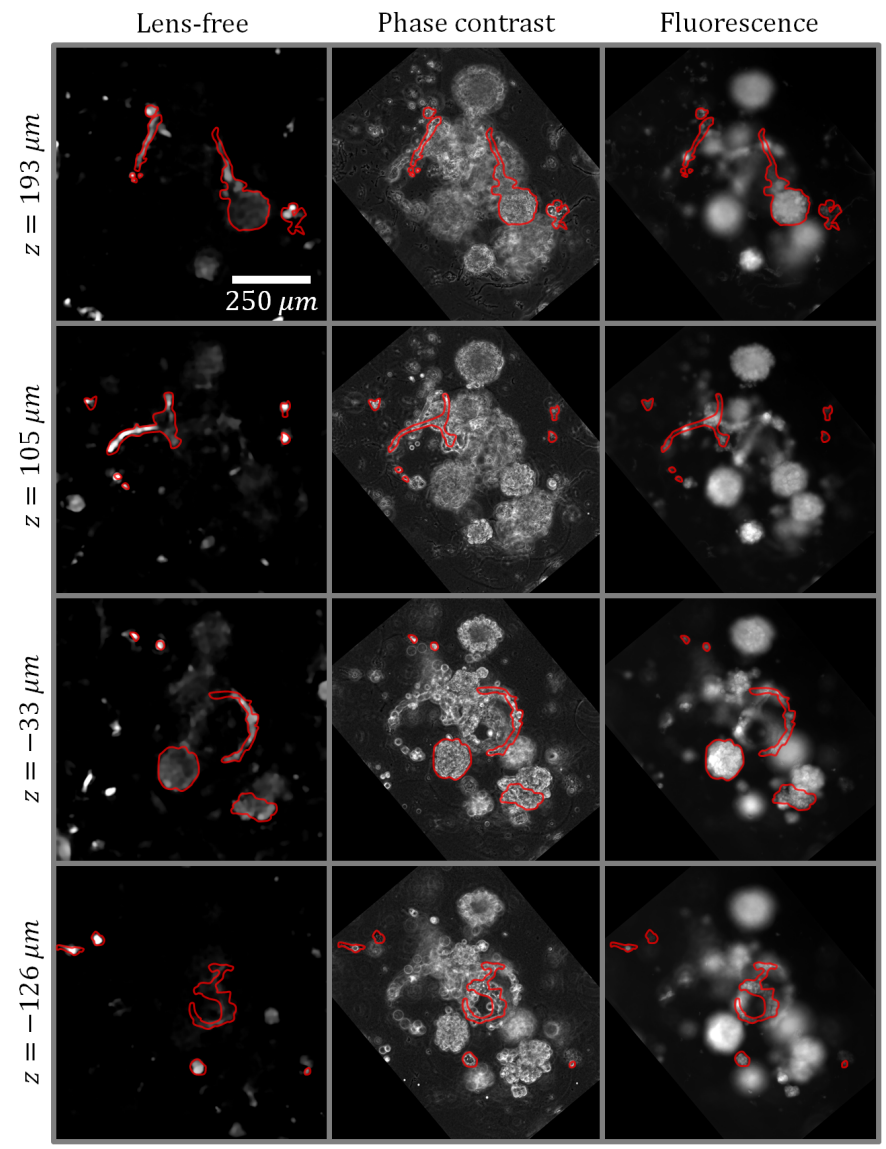

Fig. 10. Comparison of the fully 3D reconstructed volume (left) with the microscope views of phase contrast imaging (middle) and CellTracker ${ }^{\mathrm{TM}}$ visualisation (right). Different slices at different depths are presented (see visualisation 6 for a more detailed view). At each focusing distance, the main characteristic structures that are in focus on the phase contrast images were manually segmented. The red outlines were superimposed on the reconstructed slices and on the CellTracker ${ }^{\mathrm{TM}}$ visualisation.

As mentioned for method C.2, the decreasing rate of the cost function sharply diminishes after the $50^{\text {th }}$ iteration. It would have been possible to stop the algorithm at that point without degrading the reconstruction quality, leading to a reconstruction time divided by 2 .

The computational time of the $3 \mathrm{D}$ inverse problem method C.3 is its main pitfall. We saw that is mainly divided between the 3D iterative problem and the data refinement.

For the first part, as regards the iterative code itself, the bottleneck is the computation of the data fidelity term. At each iteration this step can take several minutes since it requires 3D and 2D Fourier transforms and extraction of spherical caps in the Fourier domain. In the realm of tomographic diffractive microscopy, some works have shown that an intelligent use of GPU programming can dramatically reduce this step to just a few seconds [29]. In the case of the RWPE1 reconstruction (section 3.B) this would represent a gain of $\sim 10 h$ on the $\sim 18 h$ dedicated to the iterative process.

Concerning the second part, the alignment steps were performed sequentially. Since then, we implemented a multithreaded solution aligning the data by batches in parallel. In our case, the registration process is directly divided by 12 , our number of cores. In the case of the RWPE1 reconstruction (section 3.B) the time dedicated to the alignment process would decrease from $\sim 12 h$ to $\sim 1 h$. Furthermore, the alignment method is based on the minimisation of the Least Squares between the simulated data and the experimental intensities. This solution has the advantage to work out the relative angle but can be very time consuming. In the refinement step, the simulated and experimental data are already relatively close and other faster alignment techniques may be implemented."

Another track for improvement concerns the choice of the hyper-parameters. In this work, they were empirically chosen for both methods C.2 and C.3 to provide reasonable results. A more thorough study of their effects and an optimised choice, maybe based on simulations, could also improve the quality of the reconstruction. It may also influence the large standard deviation of the background signal mentioned above: further studies are necessary to determine if it comes from the method and if a better choice for the hyper-parameters would solve the issue or if the area is not as empty as it seems. Indeed the presented cultures were grown in Matrigel ${ }^{\circledR}$. It presents good optical properties for standard microscopy $[30,31]$. but can be a very heterogeneous structure with a possible formation of fibrils or agglomerates, not adapted to diffractive microscopy. Some other extracellular matrices could be tested [32]. The optimisation of the hyper-parameters and the extracellular matrix represents an extensive study which will be subject of future work.

Finally, we showed that the sectioning capabilities outclass the focused/unfocused estimation of 2D standard microscopes. But to better quantify the resolution on the z-axis, comparisons with classical optical 3D microscopes such as confocal or lightsheet microscopes are needed .

For biological applications, it would be interesting to test the limitation of the proposed tool with stained or pigmented cells. Indeed, the reconstruction algorithms are based on the assumption that the sample is transparent. Nonetheless staining is often used in biology to label specific cells or structures. Some works have shown the possibility to use lens-free devices to image such 2D samples [33], a need which will also appear in 3D samples.

In addition, an adaptation of the device to incubator conditions would open the field of time-lapse microscopy to large volumes analysis of overall sparse 3D cell cultures, dedicated to screening assays for example.

\section{ACKNOWLEDGEMENT}

The authors would like to thank Romaric Vincent for critical reading of the manuscript.

We are grateful to Mathilde Menneteau and Stéphanie Porte who provided the biological cultures.

We would also like to thank Eric Thiébaut for his OptimPack library $^{7}$, a C implementation of the VMLM-B algorithm and also for his implementation to compute the regularisation term $\|\nabla \cdot\|_{L_{1}, \epsilon}$ for 3D objects that we adapted for complex variables. We also thank Hervé Carfantan who provided an interface ${ }^{8}$ of this library running under Matlab ${ }^{\circledR}$.

\section{REFERENCES}

1. N. Scherf and J. J. Huisken, "The smart and gentle microscope," Nature Biotechnology 33, 815-818 (2015).

${ }^{7}$ https://cral.univ-lyon1.fr/labo/perso/eric.thiebaut/?Software/OptimPack

${ }^{8}$ http://userpages.irap.omp.eu/ hcarfantan/ 
2. S. V. Kesavan, M. Momey, O. Cioni, B. David-Watine, N. Dubrulle, S. Shorte, E. Sulpice, D. Freida, B. Chalmond, J. M. Dinten, X. Gidrol, and $\mathrm{C}$. Allier, "High-throughput monitoring of major cell functions by means of lensfree video microscopy," Scientific Reports 4 (2014).

3. I. Pushkarsky, Y. Liu, W. Weaver, T. W. Su, O. Mudanyali, A. Ozcan, and D. Di Carlo, "Automated single-cell motility analysis on a chip using lensfree microscopy," Scientific Reports 4 (2014).

4. Z. Zheng, S. A. Lee, Y. Antebi, M. B. Elowitz, and C. Yang, "The ePetri dish, an on-chip cell imaging platform based on subpixel perspective sweeping microscopy (SPSM)," Proceedings of the National Academy of Sciences, U.S.A 108, 16889-16894 (2011).

5. F. Pampaloni, E. G. Reynaud, and E. H. K. Stelzer, "The third dimension bridges the gap between cell culture and live tissue," Nature Publishing Group 8, 839-845 (2007).

6. K. A. Fitzgerald, M. Malhotra, C. M. Curtin, F. J. O. Brien, and C. M. O. Driscoll, "Life in $3 \mathrm{~d}$ is never flat: $3 \mathrm{~d}$ models to optimise drug delivery," Journal of Controlled Release 215, $39-54$ (2015).

7. A. Fatehullah, S. Tan, and N. Barker, "Organoids as an in vitro model of human development and disease." Nature Cell Biology 18, 246-54 (2016).

8. M. E. Dolega, C. Allier, S. V. Kesavan, S. Gerbaud, F. Kermarrec, P. Marcoux, J.-M. Dinten, X. Gidrol, and N. Picollet-D'Hahan, "Label-free analysis of prostate acini-like 3D structures by lensfree imaging," Biosensors and Bioelectronics 49, $176-183$ (2013).

9. S. O. Isikman, W. Bishara, S. Mavandadi, F. W. Yu, S. Feng, R. Lau, and A. Ozcan, "Lens-free optical tomographic microscope with a large imaging volume on a chip," Proceedings of the National Academy of Sciences 108, 7296-7301 (2011)

10. C. Zuo, J. Sun, J. Zhang, Y. Hu, and Q. Chen, "Lensless phase microscopy and diffraction tomography with multi-angle and multiwavelength illuminations using a led matrix," Optics Express 23, 1431414328 (2015)

11. F. Momey, A. Berdeu, T. Bordy, J.-M. Dinten, F. K. Marcel, N. PicolletD'hahan, X. Gidrol, and C. Allier, "Lensfree diffractive tomography for the imaging of 3D cell cultures," Biomedical Optics Express 7, 949-962 (2016).

12. E. Wolf, "Three-dimensional structure determination of semitransparent objects from holographic data," Optics Communications 1, 153-156 (1969).

13. Y. Sung, W. Choi, C. Fang-Yen, K. Badizadegan, R. R. Dasari, and M. S. Feld, "Optical diffraction tomography for high resolution live cell imaging," Optics Express 17, 266-277 (2009).

14. O. Haeberlé, K. Belkebir, H. Giovaninni, and A. Sentenac, "Tomographic diffractive microscopy: basics, techniques and perspectives," Journal of Modern Optics 57, 686-699 (2010).

15. Y. Cotte, F. Toy, P. Jourdain, N. Pavillon, D. Boss, P. Magistretti, P. Marquet, and C. Depeursinge, "Marker-free phase nanoscopy," Nature Photonics 7, 113-117 (2013).

16. D. Gabor, "A new microscopic principle," Nature 161, 777-778 (1948).

17. M. Debailleul, B. Simon, V. Georges, O. Haeberlé, and V. Lauer, "Holographic microscopy and diffractive microtomography of transparent samples," Measurement Science and Technology 19, 074009 (2008).

18. B. Simon, M. Debailleul, A. Beghin, Y. Tourneur, and O. Haeberlé, "Highresolution tomographic diffractive microscopy of biological samples," Journal of Biophotonics 3, 462-467 (2010).

19. X. J. Liang, A. Q. Liu, C. S. Lim, T. C. Ayi, and P. H. Yap, "Determining refractive index of single living cell using an integrated microchip," Sensors and Actuators A: Physical 133, 349 - 354 (2007).

20. V. Lauer, "New approach to optical diffraction tomography yielding a vector equation of diffraction tomography and a novel tomographic microscope," Journal of Microscopy pp. 165-176 (2002).

21. J. W. Goodman, Introduction to Fourier optics (Roberts, Englewood (Colorado), 2005).

22. D. L. Donoho, “Compressed sensing," IEEE Trans. Inf. Theor. 52, 1289 1306 (2006).

23. L. Denis, D. Lorenz, E. Thiébaut, C. Fournier, and D. Trede, "Inline hologram reconstruction with sparsity constraints," Optics Letters $\mathbf{3 4}$, 3475-3477 (2009).
24. L. I. Rudin, S. Osher, and E. Fatemi, "Nonlinear total variation based noise removal algorithms," Journal of Physics D 60, 259-268 (1992).

25. P. Charbonnier, L. Blanc-Feraud, G. Aubert, and M. Barlaud, "Deterministic edge-preserving regularization in computed imaging," Transactions on Image Processing 6, 298-311 (1997).

26. J. Nocedal, "Updating quasi-newton matrices with limited storage," Mathematics of Computation 35, 773-782 (1980).

27. M. E. Dolega, F. Abeille, N. Picollet-D'hahan, and X. Gidrol, "Controlled 3D culture in matrigel microbeads to analyze clonal acinar development," Biomaterials 52, $347-357$ (2015).

28. G.-H. Chen, J. Tang, and S. Leng, "Prior image constrained compressed sensing (piccs): A method to accurately reconstruct dynamic ct images from highly undersampled projection data sets," Medical Physics 35, 660-663 (2008).

29. J. Bailleul, B. Simon, M. Debailleul, H. Liu, and O. Haeberlé, "GPU acceleration towards real-time image reconstruction in 3d tomographic diffractive microscopy," in "Real-Time Image and Video Processing 2012, Brussels, Belgium, April 19, 2012," (2012), p. 843707.

30. X. Liang, B. W. Graf, and S. A. Boppart, "Imaging engineered tissues using structural and functional optical coherence tomography," Journal of Biophotonics 2, 643-655 (2009).

31. B. W. Graf and S. A. Boppart, "Imaging and analysis of threedimensional cell culture models," Methods in Molecular Biology 591, 211-227 (2010).

32. S. C. Neves, D. B. Gomes, A. Sousa, S. J. Bidarra, P. Petrini, L. Moroni, C. C. Barrias, and P. L. Granja, "Biofunctionalized pectin hydrogels as 3d cellular microenvironments," Journal of Materials Chemistry B $\mathbf{3}$, 2096-2108 (2015).

33. A. Greenbaum, Y. Zhang, A. Feizi, P.-L. Chung, W. Luo, S. R. Kandukuri, and A. Ozcan, "Wide-field computational imaging of pathology slides using lens-free on-chip microscopy," Science Translational Medicine 6 , 267-175 (2014). 